\title{
Dynamically induced doublon repulsion in the Fermi-Hubbard model probed by a single-particle density of states
}

\author{
V. N. Valmispild $\odot,{ }^{1,2,3}$ C. Dutreix $\odot,{ }^{4}$ M. Eckstein, ${ }^{5}$ M. I. Katsnelson $\odot,{ }^{6}$ A. I. Lichtenstein, ${ }^{1,2,3}$ and E. A. Stepanov ${ }^{1}$ \\ ${ }^{1}$ I. Institute of Theoretical Physics, University of Hamburg, Jungiusstrasse 9, D-20355 Hamburg, Germany \\ ${ }^{2}$ The Hamburg Centre for Ultrafast Imaging, Luruper Chaussee 149, D-22761 Hamburg, Germany \\ ${ }^{3}$ European XFEL, Holzkoppel 4, D-22869 Schenefeld, Germany \\ ${ }^{4}$ Université Bordeaux, CNRS, LOMA, UMR 5798, F-33400 Talence, France \\ ${ }^{5}$ Department of Physics, University of Erlangen-Nuremberg, D-91058 Erlangen, Germany \\ ${ }^{6}$ Radboud University, Institute for Molecules and Materials, NL-6525AJ Nijmegen, The Netherlands
}

(Received 26 May 2020; revised 11 November 2020; accepted 12 November 2020; published 2 December 2020)

\begin{abstract}
We investigate the possibility to control dynamically the interactions between repulsively bound pairs of fermions (doublons) in correlated systems with off-resonant ac fields. We introduce an effective Hamiltonian that describes the physics of doublons up to second order in the high-frequency limit. It unveils that the doublon interaction, which is attractive in equilibrium, can be completely suppressed and then switched to repulsive by varying the power of the ac field. We show that the signature of the dynamical repulsion between doublons can be found in the single-fermion density of states averaged in time. Our results are further supported by nonequilibrium dynamical mean-field theory simulations for the half-filled Fermi-Hubbard model.
\end{abstract}

DOI: 10.1103/PhysRevB.102.220301

The ability to control matter by strong laser pulses has always intrigued researchers in many areas of physics. The recent development of femtosecond laser sources allows one to perform experiments on a timescale of the dominant microscopic interactions in materials. These experiments offer an outstanding possibility to selectively excite different collective modes, which has led to intriguing results, such as light-induced magnetism [1-8], superconductivity $[9,10]$, and topological states of matter [11-16]. The investigation of field-driven effects in fermionic systems is also motivated by inspiring results of ultracold atom physics [17-19], where the effect of the applied perturbation can be mimicked by a modulation of the position (shaking) of the lattice [20-24], or through the engineering of photon-assisted hopping amplitudes $[19,25]$.

Among different light-induced collective excitations, a large amount of attention of experimental [26-29] and theoretical [30-38] condensed matter physics is devoted to repulsively bound pairs of fermions that occupy the same lattice site. Effects related to these composite bosonic objects, known as doublons, are also actively discussed in the context of cold atoms [17,18,39-43]. Interestingly, the concept of doublons has been introduced as early as in the 1930s, within the so-called "polar model" [44] (for a more modern presentation, see Refs. $[45,46])$. Theoretically, the dynamics of doublons can be studied in the Mott-insulating regime of the Fermi-Hubbard model, where these bosonic quasiparticles have an exponentially large lifetime due to a strong repulsive on-site Coulomb interaction [47-49]. In this case, a fingerprint of doublon excitations is contained in a fermion density of states (DOS), where states related to doubly occupied lattice sites form upper and lower Hubbard subbands. The latter can be efficiently observed in (inverse) photoemission spectroscopy experiments [50-52].

One of the main interests in doublons is associated with the effect of Bose-Einstein condensation (BEC) [40,53-56] and a phase transition from an insulating to a superfluid state $[57,58]$. In equilibrium, the interaction between doublons is attractive [59-62]. Since the pioneering work by Valatin and Butler [63], it is known that the Bose gas with attractive interactions has a tendency to a phase separation (see also Refs. [64-67]). However, the BEC can be achieved introducing a short-range repulsion in the system [68-70]. Thus, the dynamical control of the doublon interaction can completely change the properties of the system and may allow for the precise control of these effects. For example, it has been shown that the local Coulomb interaction in the FermiHubbard model can be effectively switched from repulsive to attractive by applying a periodic perturbation. This can be achieved by creating a population inversion in electronic bands through a sign change of the hopping amplitude [71,72], or by a properly chosen pulse shape [73]. Later, this result has been used to modify an effective interaction between doublons, which resulted in the change of the superfluidity pairing from $s$ wave to $\eta$ pairing [74-77].

Here, we propose a different nonequilibrium mechanism to switch the doublon interaction from attractive to repulsive with an ac field. We derive an effective time-independent Hamiltonian that describes the doublon physics up to second order in the high-frequency limit of the field. It reveals that, in contrast to the works mentioned above, the repulsion between doublons is induced without a population inversion and also for considerably smaller powers of the field. Importantly, we argue that this interaction switch can be detected experimen- 
tally in a simple way via the single-fermion density of states (DOS) averaged in time. Such an observable does not require the use of time-resolved techniques and, therefore, could be routinely measured in experiments. We further support these findings numerically with nonequilibrium dynamical meanfield theory (DMFT) simulations [78]. Our result suggests that for the detection of Floquet interactions in solids the doublon channel may be a good alternative to the spin exchange interaction. The control of the spin exchange has been demonstrated in cold atoms [79], but a measurement in the solid would require the use of a much more complicated time-resolved resonant inelastic x-ray scattering technique. On the contrary, the DOS can be measured even when the system is highly excited by photodoping, which suppresses spin correlations.

High-frequency doublon Hamiltonian. We consider the time-periodic Fermi-Hubbard Hamiltonian on a square lattice,

$$
H=\sum_{\langle i j\rangle, \sigma} t_{i j}(\tau) c_{i \sigma}^{\dagger} c_{j \sigma}+U \sum_{i} n_{i \uparrow} n_{i \downarrow} .
$$

Operator $c_{i \sigma}$ annihilates an electron on site $i$ with spin $\sigma$, $n_{i \sigma}=c_{i \sigma}^{\dagger} c_{i \sigma}$ is the electron number operator, and $U$ the repulsive on-site Coulomb potential. The time periodicity arises from a uniform ac perturbation of frequency $\Omega$, directed along the square lattice diagonal $\mathbf{e}=\{1,1\}$, as implemented, for example, with an electric field driving the electrons of a material, or a shaken lattice of cold atoms [20-24]. The field is incorporated via a vector potential $\mathbf{A}(\tau)=A \mathbf{e} \cos (\tau)$, where time $\tau$ is given in units of $\Omega^{-1}$. The hopping amplitude between nearest neighbors $\langle i j\rangle$ then accumulates a Peierls phase and satisfies $t_{i j}(\tau)=t e^{-i \mathbf{A}(\tau) \cdot \mathbf{R}_{i j}}$, where $\mathbf{R}_{i j}$ is the unit real-space vector between neighboring sites.

In equilibrium, the Fermi-Hubbard Hamiltonian (1) maps via a Schrieffer-Wolff transformation onto an effective model that describes the low-energy physics of doublons in the limit $U \gg t$ [59-62]. The presence of an external time-dependent field complicates this task. So, we first perform a Magnuslike expansion in the spirit of Refs. [14,15,74,76,80-83]. This allows us to derive an effective time-independent Hamiltonian that captures the field renormalization of the fermion hopping and interaction up to the second order in the high-frequency $\operatorname{limit} U \ll \Omega$,

$$
\begin{aligned}
H^{\prime}(A)= & \sum_{\langle i j\rangle, \sigma} t^{\prime}(A) c_{i \sigma}^{\dagger} c_{j \sigma}+U^{\prime}(A) \sum_{i} n_{i \uparrow} n_{i \downarrow} \\
& +\sum_{\langle i j\rangle}\left(J^{\prime}(A) d_{i}^{\dagger} d_{j}+\frac{1}{2} V^{\prime}(A) n_{i} n_{j}+\frac{1}{2} \mathcal{I}^{\prime}(A) \mathbf{S}_{i} \mathbf{S}_{j}\right) .
\end{aligned}
$$

Here, the electron hopping $t^{\prime}(A)$ and the local Coulomb interaction $U^{\prime}(A)$ explicitly depend on the amplitude of the applied field through $A$. In addition, the high-frequency field induces purely nonequilibrium two-particle processes described by the nonlocal Coulomb potential $V^{\prime}(A)$, exchange interaction strength $I^{\prime}(A)$, and doublon hopping amplitude $J^{\prime}(A)$, for which we introduced the doublon operator $d_{j}=c_{j \downarrow} c_{j \uparrow}$ that annihilates a pair of fermions on site $j$. Their explicit expressions are in the Supplemental Material (SM) [84]. Local charge and spin densities are defined as $n_{i}=\sum_{\sigma} n_{i \sigma}$ and

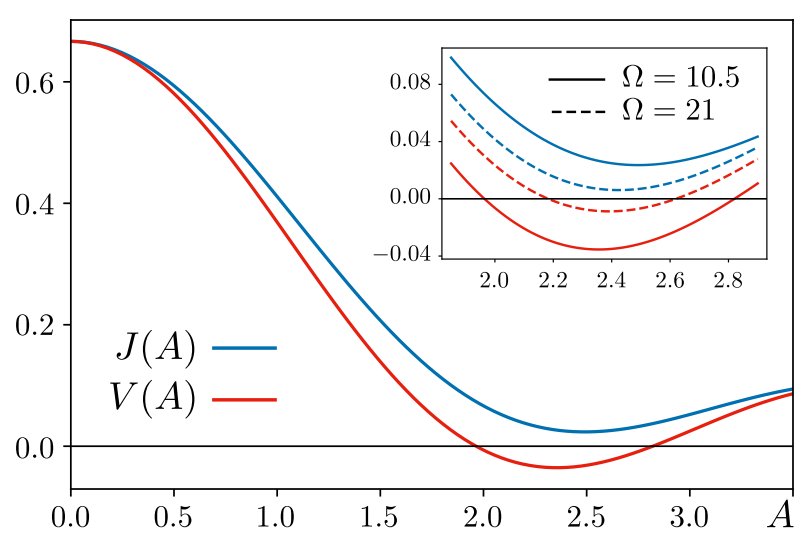

FIG. 1. Doublon parameters of the effective Hamiltonian (3) as a function of the field strength $A$ for $\Omega=10.5$ and $U=3$. The inset shows a field range for both frequencies $\Omega=10.5$ and $\Omega=21$ in which the nonlocal doublon interaction $V$ changes signs, whereas the doublon hopping amplitude $J$ does not.

$\mathbf{S}_{i}=\frac{1}{2} \sum_{\sigma, \sigma^{\prime}} c_{i \sigma}^{\dagger} \boldsymbol{\sigma}_{\sigma \sigma^{\prime}} c_{i \sigma^{\prime}}$, respectively. $\sigma=\left\{\sigma^{x}, \sigma^{y}, \sigma^{z}\right\}$ is a vector of Pauli matrices.

Single-particle hopping processes that change the number of doubly occupied sites also change the total energy of the system. Here, we focus on the low-energy physics of doublons and disregard such processes in the Schrieffer-Wolff transformation of Hamiltonian (2). This leads to an effective Hamiltonian that describes the doublon subsystem in the nonequilibrium steady state,

$$
H^{d}(A)=\sum_{\langle i j\rangle} J(A) d_{i}^{\dagger} d_{j}-\sum_{\langle i j\rangle} V(A) \rho_{i} \rho_{j},
$$

where $\rho_{i}=d_{i}^{\dagger} d_{i}=n_{i \uparrow} n_{i \downarrow}$ is the local double occupancy operator. The hopping amplitude $J(A)$ and nonlocal interaction potential $V(A)$ of doublons depend on the strength of the external field as [84]

$$
\begin{gathered}
J(A)=\frac{2 t^{2}}{U}\left(\mathcal{J}_{0}^{2}(A)-\frac{2 U^{2}}{\Omega^{2}} \sum_{m>0} \frac{(-1)^{m}}{m^{2}} \mathcal{J}_{m}^{2}(A)\right), \\
V(A)=\frac{2 t^{2}}{U}\left(\mathcal{J}_{0}^{2}(A)-\frac{2 U^{2}}{\Omega^{2}} \sum_{m>0} \frac{1}{m^{2}} \mathcal{J}_{m}^{2}(A)\right),
\end{gathered}
$$

where $\mathcal{J}_{m}(A)$ is the $m$ th-order Bessel function of the first kind. The zeroth-order contribution in the limit $U \ll \Omega$ renormalizes the doublon hopping and interaction in the same way, $J(A)=V(A)=\mathcal{J}_{0}^{2}(A) 2 t^{2} / U+O(U / \Omega)$. As noticed in Ref. [74], the field-dependent factor $\mathcal{J}_{0}^{2}(A)$ simply acts as an overall scaling parameter and results in trivial physics. Thus, the doublon hopping and interaction both remain positive, as in equilibrium. Here, we go further and consider the secondorder contributions in the high-frequency limit [84]. We find that these contributions of order $U^{2} / \Omega^{2}$ in Eqs. (4) and (5) now lift the degeneracy between $J$ and $V$. In particular, this effect becomes important for field amplitudes near $A \simeq 2.4$, where the zeroth-order contribution vanishes. Figure 1 shows that this even allows the doublon interaction $V$ to become repulsive out of equilibrium, while the hopping amplitude $J$ does not change sign. This shows that an independent control of $V$ and $J$ is not only possible in the vicinity of the resonance 


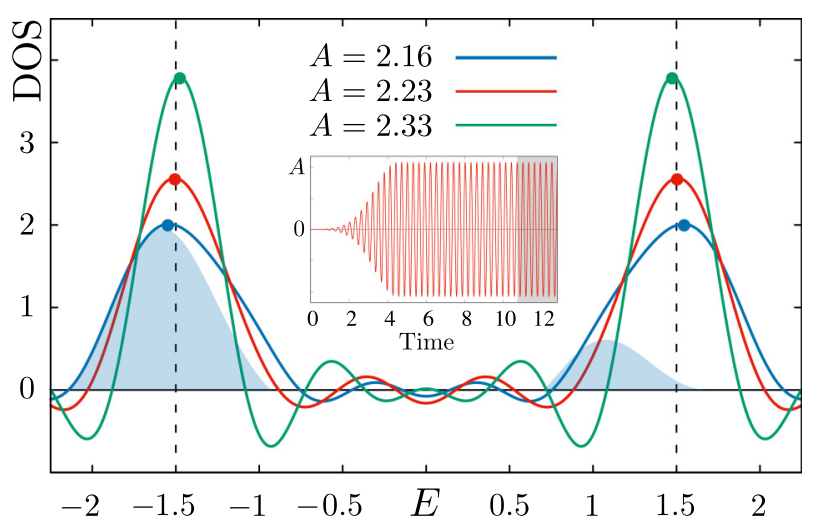

FIG. 2. Time-averaged fermionic DOS obtained for $\Omega=21$ and various field strengths $A=2.16$ (blue), $A=2.23$ (red), and $A=2.33$ (green). Colored points mark the top of the Hubbard subbands. Vertical dashed lines at $E= \pm U / 2= \pm 1.5$ are a guide for the eyes. Shaded blue areas indicate the time-averaged population of the upper and lower subbands at the field $A=2.16$. The inset shows the time profile of the vector potential. The time averaging is performed over the last eight periods highlighted in gray.

$\Omega=U$ [74], but also in the high-frequency limit (where energy absorption is well controlled).

Nonequilibrium Hubbard subbands. The switch of the doublon interaction may in principle be observed in experiment through a measurement of the doublon-doublon susceptibility. However, the latter is hardly accessible, as it corresponds to a four-fermion response function. Instead, we now show that signatures of the doublon interaction can also be revealed through the single-fermion density of states (DOS), as routinely measured in experiments [50-52]. To illustrate this point, we perform time-dependent DMFT simulations for the half-filled Hubbard model (1) [33]. We consider the local Coulomb interaction $U=3$, so that the driven system for the range of fields $A$ when the doublon interaction $V(A)$ changes sign lies in a Mott-insulating state. Values $\Omega=10.5$ and $\Omega=21$ for the frequency of the field are taken to justify the requirement $(U / \Omega)^{2} \ll 1$ for the high-frequency expansion. The energy is given in units of the electron hopping amplitude $t$. The ac field is turned on up to the maximum value $A_{\max }=\sqrt{2} A$ following the exponential ramp $\exp \left\{-\left(t-t_{0}\right)^{2} /\left(2 \sigma^{2}\right)\right\}$ in order to avoid heating $[72,85]$. Here, $\sigma=\frac{d}{2 \sqrt{2 \ln 2}}$ and $d$ is a full width at half maximum of a pulse. The time profile of the field is shown in the inset of Fig. 2. We then determine the fermion DOS given by the spectral function $A^{R}(t, E)$ defined as (see, e.g., Ref. [86])

$$
A^{\alpha}(t, E)=-\frac{1}{\pi} \operatorname{Im} \int_{0}^{s_{\max }} d s e^{i E s} G_{\mathrm{loc}}^{\alpha}(t, t-s)
$$

where for $G_{\mathrm{loc}}^{\alpha}\left(t, t^{\prime}\right)$ we take the local retarded Green's function $G_{\mathrm{loc}}^{R}\left(t, t^{\prime}\right)=-i \theta\left(t-t^{\prime}\right)\left\langle\left\{c_{i}(t), c_{i}^{\dagger}\left(t^{\prime}\right)\right\}\right\rangle$. We perform the numerical time-dependent DMFT calculations within the iterative perturbation theory (IPT) [86] on a $32 \times 32 k$ grid starting with the inverse temperature $\beta=5$. We finally average over the last eight periods of time (gray area in the inset of Fig. 2), for which the behavior of time-resolved observables, such as the double occupancy presented in Fig. 4 (left), indi-

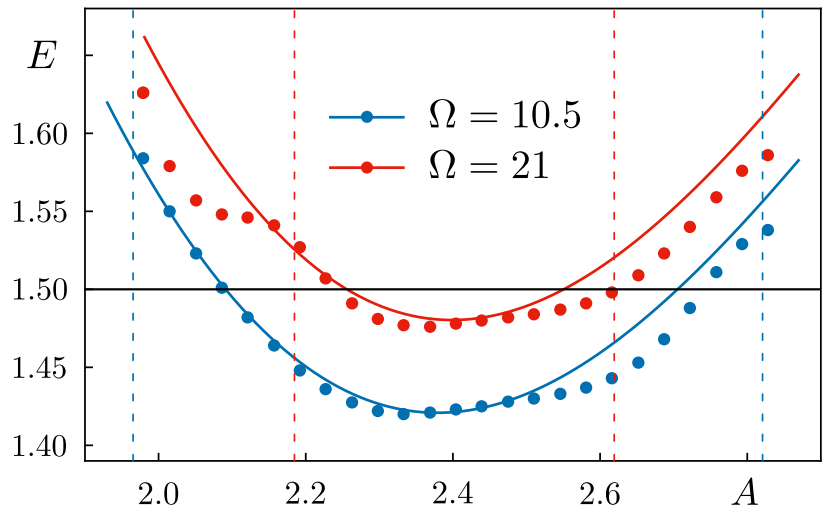

FIG. 3. Position of the top of the upper Hubbard subband as a function of the applied light $A$. Results are obtained for two frequencies $\Omega=10.5$ (blue color) and $\Omega=21$ (red color). Solid lines correspond to the estimation $U_{\text {eff }} / 2$ obtained from the effective local Coulomb interaction. Points correspond to the nonequilibrium DMFT result. Vertical dashed lines indicate fields at which the doublon-doublon interaction $V(A)$ changes sign. The horizontal line $E=U / 2=1.5$ serves as a guide for the eyes.

cates that the system is in a nonequilibrium steady state. This will allow us to compare the numerical simulations with the effective time-independent description in Eq. (2).

Figure 2 shows the single-fermion DOS resulting from the nonequilibrium DMFT simulations for $\Omega=21$ and various field amplitudes. The two spectral peaks below and above the Fermi energy $(E=0)$ are the lower and upper Hubbard subbands that correspond to doublon and holon (fully unoccupied site) states, respectively. This structure of the DOS confirms that, for the range of field amplitudes we consider, the interacting fermion system is indeed a Mott insulator. An occupation function can be obtained as a time-averaged spectral function $A^{<}(t, E)$ (6) of the lesser Green's function $G_{\mathrm{loc}}^{<}\left(t, t^{\prime}\right)=i\left\langle c_{i}^{\dagger}\left(t^{\prime}\right) c_{i}(t)\right\rangle$. The shaded blue areas in Fig. 2 show that the upper Hubbard subband is only slightly populated upon driving. An effective temperature of the system can be estimated from the nonequilibrium distribution function $A^{<}(t, E) / A^{R}(t, E)$, and gives an effective inverse temperature of order $\beta=2$.

We find that varying the field amplitude results in the energy shift of the Hubbard subbands. It is not surprising, since the position of the subbands is determined by the local Coulomb interaction as $E \simeq \pm U^{\prime}(A) / 2$, which explicitly depends on the field amplitude. Points in Fig. 3 represent the peak energy of the upper Hubbard subband as a function of $A$ obtained from nonequilibrium DMFT simulations for two different frequencies. While $A$ increases, we observe that the subbands first move closer to the Fermi level as if they attract each other. Above a critical field, the interaction between peaks switches to repulsive, and the distance between them increases again. As we now show, this behavior of Hubbard subbands is a manifestation of an attractive-repulsive transition of the interaction between doublons.

Detection of repulsive doublon interactions. We find that the local Coulomb potential $U^{\prime}(A)$ alone cannot explain the behavior of Hubbard subbands obtained from DMFT 


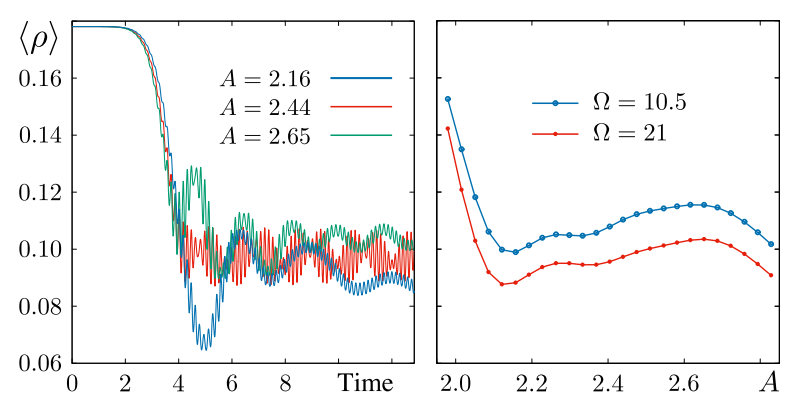

FIG. 4. Left: Doublon density as a function of time obtained for three different values of the vector potential $A=2.16$ (blue line), $A=2.44$ (red line), and $A=2.65$ (green line). Right: Time-averaged doublon density as a function of vector potential obtained for two frequencies $\Omega=10.5$ (blue line) and $\Omega=21$ (red line).

simulations in Fig. 3. As shown in the SM [84], $U^{\prime}(A)<U$ for any field amplitude $A$. Then, the peak of the upper Hubbard subband, if given by $U^{\prime}(A) / 2$, cannot exceed the equilibrium value of $U / 2$. For this reason, we further account for the effects of the nonlocal interactions and hopping processes in Hamiltonian (2) on the position of the subbands. We first map the nonlocal Coulomb potential onto an on-site potential through the Peierls-Feynman-Bogoliubov variational principle [87-89]. This leads to the local Coulomb interaction $U^{*}(A)=U^{\prime}(A)-V^{\prime}(A)$ [90]. The effect of other terms in Hamiltonian (2) can be taken into account perturbatively. In particular, we find that only the two-hopping processes contribute to the effective local Coulomb potential $U_{\text {eff }}$ [84]. This finally results in

$$
U_{\mathrm{eff}}(A)=U+\frac{2 t^{2}}{U}\left(\frac{1}{\langle\rho\rangle} \mathcal{J}_{0}^{2}(A)-\frac{9 U^{2}}{\Omega^{2}} \sum_{m>0} \frac{1}{m^{2}} \mathcal{J}_{m}^{2}(A)\right) .
$$

Thus, the behavior of Hubbard subbands as the function of field can be approximated by the following relation: $E=U_{\text {eff }}(A) / 2$. The mean value of the double occupancy $\langle\rho\rangle$ that enters Eq. (7) can be extracted from Fig. 4, which represents the nonequilibrium DMFT result. For two different frequencies $\Omega=10.5$ and $\Omega=21$ we find $\langle\rho\rangle \simeq 0.11$ and $\langle\rho\rangle \simeq 0.10$, respectively. Remarkably, the solid lines in Fig. 3 show that this simple estimation for the position of the subbands based on the effective time-independent description of the problem (2) accurately reproduces the result of nonequilibrium DMFT simulations for the initial time-dependent model (1). This fact suggests that the introduced Hamiltonian (2) correctly describes properties of the nonequilibrium steady state of the system. In addition, the relation (7) can also serve as the measure for the average double occupancy of the lattice site $\langle\rho\rangle$.

We can further relate the effective local Coulomb potential $U_{\text {eff }}$ to the doublon interaction $V(A)$ in Hamiltonian (3). In equilibrium, the mean double occupancy $\langle\rho\rangle$ per lattice site does not exceed $1 / 4$ at half filling. Larger values of $\langle\rho\rangle$ indicate a population inversion. This can occur out of equilibrium but for much stronger fields and smaller frequencies of the field $(\Omega \sim U$ close to a resonant driving between Hubbard subbands) than the ones we are dealing with here $[71,76,91]$.
In our case, Fig. 4 shows that the double occupancy does not exceed $1 / 4$. Thus, the attraction-repulsion transition of doublon interactions in Fig. 1 does not involve any population inversion, in contrast to a previous proposal [73]. Besides, if we consider the maximum value of the mean double occupancy, i.e., $\langle\rho\rangle=1 / 4$, the energy shift of the Hubbard subbands with respect to their equilibrium position in the atomic limit $( \pm U / 2)$ is

$$
\Delta(A)=\left[U_{\text {eff }}(A)-U\right] / 2 \simeq 2 V(A) .
$$

Therefore, this shift $\Delta$ is a single-fermion measurement of the strength of the doublon-doublon interaction $V(A)$ for $\langle\rho\rangle=1 / 4$. For smaller values of $\langle\rho\rangle$, we more generally find $\Delta(A) \geqslant 2 V(A)$. It follows that the negative value of $\Delta(A)<0$ obtained in the nonequilibrium DMFT simulations in Fig. 3 is an indirect indication that the doublon interaction, initially attractive at zero field, has become repulsive out of equilibrium. The value of the field at which the interaction between doublons changes sign is depicted in Fig. 3 by vertical dashed lines. Importantly, the total suppression of the doublon interaction $V(A)=0$ happens at a considerably smaller power of the field $A$ compared to the regime of dynamical localization, determined by the first root of the Bessel function $\mathcal{J}_{0}(A)=0$. Therefore, the high-frequency driving provides a unique possibility to explore the regime of a weakly interacting doublon liquid, whereas in equilibrium doublons are strongly interacting $J(0)=V(0)$ as follows from Eqs. (4) and (5).

Conclusions. To conclude, in this Rapid Communication we have studied the effect of the applied high-frequency perturbation on the doublon subsystem of the fermion Hubbard model. First, we have introduced an effective time-independent Hamiltonian that describes a time-averaged dynamics of doublons. We have shown that the hopping amplitude and nonlocal interaction of this effective model can be controlled by the value of the vector potential. Moreover, in a certain range of fields, a possibility for a dynamical attraction-repulsion transition of doublons has been investigated. Further, we have shown that the signature of this transition can be found in the behavior of Hubbard subbands of the single-fermion density of states. We have proposed a simple explanation of the observed effect based on the renormalization of the local Coulomb potential via hopping processes and a nonlocal Coulomb interaction. The obtained result provides a clear criterion for the experimental confirmation of the repulsive interactions between doublons that involves only a local single-fermion observable.

Acknowledgments. The authors thank Michael Potthoff and Sergey Brener for useful discussions and comments. The authors acknowledge the support by the Cluster of Excellence "Advanced Imaging of Matter" of the Deutsche Forschungsgemeinschaft (DFG) - EXC 2056 - Project No. ID390715994. This research was also supported through the European XFEL and DESY computational resources in the Maxwell infrastructure operated at Deutsches Elektronen-Synchrotron (DESY), Hamburg, Germany. C.D. acknowledges the support 
of Idex Bordeaux (Maesim Risky project 2019 of the LAPHIA Program). The work of V.N.V., M.I.K., and A.I.L. is sup- ported by European Research Council via Synergy Grant No. 854843 - FASTCORR.
[1] E. Beaurepaire, J.-C. Merle, A. Daunois, and J.-Y. Bigot, Ultrafast Spin Dynamics in Ferromagnetic Nickel, Phys. Rev. Lett. 76, 4250 (1996).

[2] A. V. Kimel, A. Kirilyuk, P. A. Usachev, R. V. Pisarev, A. M. Balbashov, and T. Rasing, Ultrafast non-thermal control of magnetization by instantaneous photomagnetic pulses, Nature (London) 435, 655 (2005).

[3] A. Kirilyuk, A. V. Kimel, and T. Rasing, Ultrafast optical manipulation of magnetic order, Rev. Mod. Phys. 82, 2731 (2010).

[4] B. Koopmans, G. Malinowski, F. Dalla Longa, D. Steiauf, M. Fähnle, T. Roth, M. Cinchetti, and M. Aeschlimann, Explaining the paradoxical diversity of ultrafast laser-induced demagnetization, Nat. Mater. 9, 259 (2010).

[5] A. Melnikov, I. Razdolski, T. O. Wehling, E. Th. Papaioannou, V. Roddatis, P. Fumagalli, O. Aktsipetrov, A. I. Lichtenstein, and U. Bovensiepen, Ultrafast Transport of Laser-Excited SpinPolarized Carriers in $\mathrm{Au} / \mathrm{Fe} / \mathrm{MgO}(001)$, Phys. Rev. Lett. 107, 076601 (2011).

[6] T. Satoh, Y. Terui, R. Moriya, B. A. Ivanov, K. Ando, E. Saitoh, T. Shimura, and K. Kuroda, Directional control of spinwave emission by spatially shaped light, Nat. Photonics 6, 662 (2012).

[7] A. J. Schellekens, K. C. Kuiper, R. R. J. C. de Wit, and B. Koopmans, Ultrafast spin-transfer torque driven by femtosecond pulsed-laser excitation, Nat. Commun. 5, 4333 (2014).

[8] A. Stupakiewicz, K. Szerenos, D. Afanasiev, A. Kirilyuk, and A. V. Kimel, Ultrafast nonthermal photo-magnetic recording in a transparent medium, Nature (London) 542, 71 (2017).

[9] D. Fausti, R. I. Tobey, N. Dean, S. Kaiser, A. Dienst, M. C. Hoffmann, S. Pyon, T. Takayama, H. Takagi, and A. Cavalleri, Light-induced superconductivity in a stripe-ordered cuprate, Science 331, 189 (2011).

[10] K. Takasan, A. Daido, N. Kawakami, and Y. Yanase, Laserinduced topological superconductivity in cuprate thin films, Phys. Rev. B 95, 134508 (2017).

[11] T. Oka and H. Aoki, Photovoltaic Hall effect in graphene, Phys. Rev. B 79, 081406(R) (2009).

[12] N. H. Lindner, G. Refael, and V. Galitski, Floquet topological insulator in semiconductor quantum wells, Nat. Phys. 7, 490 (2011).

[13] A. G. Grushin, Á. Gómez-León, and T. Neupert, Floquet Fractional Chern Insulators, Phys. Rev. Lett. 112, 156801 (2014).

[14] C. Dutreix, E. A. Stepanov, and M. I. Katsnelson, Laserinduced topological transitions in phosphorene with inversion symmetry, Phys. Rev. B 93, 241404(R) (2016).

[15] E. A. Stepanov, C. Dutreix, and M. I. Katsnelson, Dynamical and Reversible Control of Topological Spin Textures, Phys. Rev. Lett. 118, 157201 (2017).

[16] J. W. McIver, B. Schulte, F.-U. Stein, T. Matsuyama, G. Jotzu, G. Meier, and A. Cavalleri, Light-induced anomalous Hall effect in graphene, Nat. Phys. 16, 38 (2020).

[17] M. Lewenstein, A. Sanpera, V. Ahufinger, B. Damski, A. Sen(De), and U. Sen, Ultracold atomic gases in optical lattices:
Mimicking condensed matter physics and beyond, Adv. Phys. 56, 243 (2007).

[18] I. Bloch, J. Dalibard, and W. Zwerger, Many-body physics with ultracold gases, Rev. Mod. Phys. 80, 885 (2008).

[19] A. Eckardt, Colloquium: Atomic quantum gases in periodically driven optical lattices, Rev. Mod. Phys. 89, 011004 (2017).

[20] H. Lignier, C. Sias, D. Ciampini, Y. Singh, A. Zenesini, O. Morsch, and E. Arimondo, Dynamical Control of Matter-Wave Tunneling in Periodic Potentials, Phys. Rev. Lett. 99, 220403 (2007).

[21] J. Struck, C. Ölschläger, M. Weinberg, P. Hauke, J. Simonet, A. Eckardt, M. Lewenstein, K. Sengstock, and P. Windpassinger, Tunable Gauge Potential for Neutral and Spinless Particles in Driven Optical Lattices, Phys. Rev. Lett. 108, 225304 (2012).

[22] P. Hauke, O. Tieleman, A. Celi, C. Ölschläger, J. Simonet, J. Struck, M. Weinberg, P. Windpassinger, K. Sengstock, M. Lewenstein, and A. Eckardt, Non-Abelian Gauge Fields and Topological Insulators in Shaken Optical Lattices, Phys. Rev. Lett. 109, 145301 (2012).

[23] M. C. Rechtsman, J. M. Zeuner, Y. Plotnik, Y. Lumer, D. Podolsky, F. Dreisow, S. Nolte, M. Segev, and A. Szameit, Photonic floquet topological insulators, Nature (London) 496, 196 (2013).

[24] G. Jotzu, M. Messer, R. Desbuquois, M. Lebrat, T. Uehlinger, D. Greif, and T. Esslinger, Experimental realization of the topological Haldane model with ultracold fermions, Nature (London) 515, 237 (2014).

[25] N. Goldman, G. Juzeliūnas, P. Öhberg, and I. B. Spielman, Light-induced gauge fields for ultracold atoms, Rep. Prog. Phys. 77, 126401 (2014).

[26] S. Iwai, M. Ono, A. Maeda, H. Matsuzaki, H. Kishida, H. Okamoto, and Y. Tokura, Ultrafast Optical Switching to a Metallic State by Photoinduced Mott Transition in a HalogenBridged Nickel-Chain Compound, Phys. Rev. Lett. 91, 057401 (2003).

[27] H. Okamoto, H. Matsuzaki, T. Wakabayashi, Y. Takahashi, and T. Hasegawa, Photoinduced Metallic State Mediated by Spin-Charge Separation in a One-Dimensional Organic Mott Insulator, Phys. Rev. Lett. 98, 037401 (2007).

[28] H. Okamoto, T. Miyagoe, K. Kobayashi, H. Uemura, H. Nishioka, H. Matsuzaki, A. Sawa, and Y. Tokura, Ultrafast charge dynamics in photoexcited $\mathrm{Nd}_{2} \mathrm{CuO}_{4}$ and $\mathrm{La}_{2} \mathrm{CuO}_{4}$ cuprate compounds investigated by femtosecond absorption spectroscopy, Phys. Rev. B 82, 060513(R) (2010).

[29] S. Wall, D. Brida, S. R. Clark, H. P. Ehrke, D. Jaksch, A. Ardavan, S. Bonora, H. Uemura, Y. Takahashi, T. Hasegawa, H. Okamoto, G. Cerullo, and A. Cavalleri, Quantum interference between charge excitation paths in a solid-state Mott insulator, Nat. Phys. 7, 114 (2011).

[30] F. Hofmann and M. Potthoff, Doublon dynamics in the extended Fermi-Hubbard model, Phys. Rev. B 85, 205127 (2012).

[31] M. Eckstein and P. Werner, Photoinduced States in a Mott Insulator, Phys. Rev. Lett. 110, 126401 (2013). 
[32] M. Eckstein and P. Werner, Dielectric breakdown of Mott insulators-doublon production and doublon heating, J. Phys.: Conf. Ser. 427, 012005 (2013).

[33] H. Aoki, N. Tsuji, M. Eckstein, M. Kollar, T. Oka, and P. Werner, Nonequilibrium dynamical mean-field theory and its applications, Rev. Mod. Phys. 86, 779 (2014).

[34] M. Eckstein and P. Werner, Ultra-fast photo-carrier relaxation in Mott insulators with short-range spin correlations, Sci. Rep. 6, 21235 (2016).

[35] Y. Wang, M. Claassen, B. Moritz, and T. P. Devereaux, Producing coherent excitations in pumped Mott antiferromagnetic insulators, Phys. Rev. B 96, 235142 (2017).

[36] R. Rausch and M. Potthoff, Filling-dependent doublon dynamics in the one-dimensional Hubbard model, Phys. Rev. B 95, 045152 (2017).

[37] M. Ligges, I. Avigo, D. Golež, H. U. R. Strand, Y. Beyazit, K. Hanff, F. Diekmann, L. Stojchevska, M. Kalläne, P. Zhou, K. Rossnagel, M. Eckstein, P. Werner, and U. Bovensiepen, Ultrafast Doublon Dynamics in Photoexcited $1 T-\mathrm{TaS}_{2}$, Phys. Rev. Lett. 120, 166401 (2018).

[38] R. Rausch and M. Potthoff, Pump-probe Auger-electron spectroscopy of Mott insulators, Phys. Rev. B 99, 205108 (2019).

[39] I. Bloch, Ultracold quantum gases in optical lattices, Nat. Phys. 1, 23 (2005).

[40] K. Winkler, G. Thalhammer, F. Lang, R. Grimm, J. Hecker Denschlag, A. J. Daley, A. Kantian, H. P. Büchler, and P. Zoller, Repulsively bound atom pairs in an optical lattice, Nature (London) 441, 853 (2006).

[41] C. Trefzger, C. Menotti, B. Capogrosso-Sansone, and M. Lewenstein, Ultracold dipolar gases in optical lattices, J. Phys. B: At., Mol. Opt. Phys. 44, 193001 (2011).

[42] Z. Xu, S. Chiesa, S. Yang, S.-Q. Su, D. E. Sheehy, J. Moreno, R. T. Scalettar, and M. Jarrell, Response to dynamical modulation of the optical lattice for fermions in the Hubbard model, Phys. Rev. A 84, 021607(R) (2011).

[43] L. W. Cheuk, M. A. Nichols, K. R. Lawrence, M. Okan, H. Zhang, E. Khatami, N. Trivedi, T. Paiva, M. Rigol, and M. W. Zwierlein, Observation of spatial charge and spin correlations in the 2D Fermi-Hubbard model, Science 353, 1260 (2016).

[44] S. Schubin and S. Wonsowsky, On the electron theory of metals, Proc. R. Soc. London, Ser. A 145, 159 (1934).

[45] S. V. Vonsovsky and M. I. Katsnelson, Some types of instabilities in the electron energy spectrum of the polar model of the crystal. I. The maximum-polarity state, J. Phys. C 12, 2043 (1979).

[46] S. V. Vonsovsky and M. I. Katsnelson, Some types of instabilities in the electron energy spectrum of the polar model of the crystal. II. The criterion of stability of a metallic state, J. Phys. C 12, 2055 (1979).

[47] A. Rosch, D. Rasch, B. Binz, and M. Vojta, Metastable Superfluidity of Repulsive Fermionic Atoms in Optical Lattices, Phys. Rev. Lett. 101, 265301 (2008).

[48] R. Sensarma, D. Pekker, A. M. Rey, M. D. Lukin, and E. Demler, Relaxation of Fermionic Excitations in a Strongly Attractive Fermi Gas in an Optical Lattice, Phys. Rev. Lett. 107, 145303 (2011).

[49] M. Eckstein and P. Werner, Thermalization of a pump-excited Mott insulator, Phys. Rev. B 84, 035122 (2011).
[50] H. Neddermeyer, in Photoemission and the Electronic Properties of Surfaces, edited by B. Feuerbacher, B. Fitton, and R. F. Willis (John Wiley \& Sons Ltd, Chichester, New York, Brisbane, Toronto, 1979), Vol. 183, p. 177.

[51] R. Courths and S. Hüfner, Photoemission experiments on copper, Phys. Rep. 112, 53 (1984).

[52] A. Damascelli, Z. Hussain, and Z.-X. Shen, Angle-resolved photoemission studies of the cuprate superconductors, Rev. Mod. Phys. 75, 473 (2003).

[53] S. Jochim, M. Bartenstein, A. Altmeyer, G. Hendl, S. Riedl, C. Chin, J. Hecker Denschlag, and R. Grimm, Bose-Einstein condensation of molecules, Science 302, 2101 (2003).

[54] M. W. Zwierlein, C. A. Stan, C. H. Schunck, S. M. F. Raupach, S. Gupta, Z. Hadzibabic, and W. Ketterle, Observation of Bose-Einstein Condensation of Molecules, Phys. Rev. Lett. 91, 250401 (2003).

[55] M. Greiner, C. A. Regal, and D. S. Jin, Emergence of a molecular Bose-Einstein condensate from a Fermi gas, Nature (London) 426, 537 (2003).

[56] D. Petrosyan, B. Schmidt, J. R. Anglin, and M. Fleischhauer, Quantum liquid of repulsively bound pairs of particles in a lattice, Phys. Rev. A 76, 033606 (2007).

[57] M. Greiner, O. Mandel, T. Esslinger, T. W. Hänsch, and I. Bloch, Quantum phase transition from a superfluid to a Mott insulator in a gas of ultracold atoms, Nature (London) 415, 39 (2002).

[58] H. Yokoyama, T. Miyagawa, and M. Ogata, Effect of doublonholon binding on Mott transition-variational Monte Carlo study of two-dimensional Bose Hubbard models, J. Phys. Soc. Jpn. 80, 084607 (2011).

[59] K. A. Chao, J. Spałek, and A. M. Oleś, Degenerate perturbation theory and its application to the Hubbard model, Phys. Lett. A 64, 163 (1977).

[60] K. A. Chao, J. Spałek, and A. M. Oleś, Kinetic exchange interaction in a narrow S-band, J. Phys. C 10, L271 (1977).

[61] A. H. MacDonald, S. M. Girvin, and D. Yoshioka, $\frac{t}{U}$ expansion for the Hubbard model, Phys. Rev. B 37, 9753 (1988).

[62] J. Spałek, $t-J$ model then and now: A personal perspective from the pioneering times, Acta Phys. Pol. A 111, 409 (2007).

[63] J. G. Valatin and D. Butler, On the collective properties of a boson system, Nuovo Cimento 10, 37 (1958).

[64] Y. Kagan, G. V. Shlyapnikov, and J. T. M. Walraven, BoseEinstein Condensation in Trapped Atomic Gases, Phys. Rev. Lett. 76, 2670 (1996).

[65] R. J. Dodd, M. Edwards, C. J. Williams, C. W. Clark, M. J. Holland, P. A. Ruprecht, and K. Burnett, Role of attractive interactions on Bose-Einstein condensation, Phys. Rev. A 54, 661 (1996).

[66] E. V. Shuryak, Metastable Bose condensate made of atoms with attractive interaction, Phys. Rev. A 54, 3151 (1996).

[67] L. P. Pitaevskii, Dynamics of collapse of a confined Bose gas, Phys. Lett. A 221, 14 (1996).

[68] P. Nozières and D. Saint James, Particle vs. pair condensation in attractive Bose liquids, J. Phys. France 43, 1133 (1982).

[69] M. J. Rice and Y. R. Wang, Superconductivity in a quasi-twodimensional Bose gas, Phys. Rev. B 37, 5893 (1988).

[70] M. Yu. Kagan and D. V. Efremov, Two-particle pairing and phase separation in a two-dimensional Bose gas with one or two sorts of bosons, Phys. Rev. B 65, 195103 (2002). 
[71] A. Rapp, S. Mandt, and A. Rosch, Equilibration Rates and Negative Absolute Temperatures for Ultracold Atoms in Optical Lattices, Phys. Rev. Lett. 105, 220405 (2010).

[72] N. Tsuji, T. Oka, P. Werner, and H. Aoki, Dynamical Band Flipping in Fermionic Lattice Systems: An ac-Field-Driven Change of the Interaction from Repulsive to Attractive, Phys. Rev. Lett. 106, 236401 (2011).

[73] N. Tsuji, T. Oka, H. Aoki, and P. Werner, Repulsion-toattraction transition in correlated electron systems triggered by a monocycle pulse, Phys. Rev. B 85, 155124 (2012).

[74] S. Kitamura and H. Aoki, $\eta$-pairing superfluid in periodicallydriven fermionic Hubbard model with strong attraction, Phys. Rev. B 94, 174503 (2016).

[75] T. Kaneko, T. Shirakawa, S. Sorella, and S. Yunoki, Photoinduced $\eta$ Pairing in the Hubbard Model, Phys. Rev. Lett. 122, 077002 (2019).

[76] F. Peronaci, O. Parcollet, and M. Schiró, Enhancement of local pairing correlations in periodically driven Mott insulators, Phys. Rev. B 101, 161101(R) (2020).

[77] J. Li, D. Golez, P. Werner, and M. Eckstein, $\eta$-paired superconducting hidden phase in photodoped Mott insulators, Phys. Rev. B 102, 165136 (2020).

[78] A. Georges, G. Kotliar, W. Krauth, and M. J. Rozenberg, Dynamical mean-field theory of strongly correlated fermion systems and the limit of infinite dimensions, Rev. Mod. Phys. 68, 13 (1996).

[79] F. Görg, M. Messer, K. Sandholzer, G. Jotzu, R. Desbuquois, and T. Esslinger, Enhancement and sign change of magnetic correlations in a driven quantum many-body system, Nature (London) 553, 481 (2018).

[80] A. P. Itin and A. I. Neishtadt, Effective Hamiltonians for fastly driven tight-binding chains, Phys. Lett. A 378, 822 (2014).
[81] A. P. Itin and M. I. Katsnelson, Effective Hamiltonians for Rapidly Driven Many-Body Lattice Systems: Induced Exchange Interactions and Density-Dependent Hoppings, Phys. Rev. Lett. 115, 075301 (2015).

[82] M. Bukov, M. Kolodrubetz, and A. Polkovnikov, SchriefferWolff Transformation for Periodically Driven Systems: Strongly Correlated Systems with Artificial Gauge Fields, Phys. Rev. Lett. 116, 125301 (2016).

[83] C. Dutreix and M. I. Katsnelson, Dynamical control of electronphonon interactions with high-frequency light, Phys. Rev. B 95, 024306 (2017).

[84] See Supplemental Material at http://link.aps.org/supplemental/ 10.1103/PhysRevB.102.220301 for the derivation of a time-independent doublon Hamiltonian and effective on-site Coulomb potential.

[85] W. W. Ho and D. A. Abanin, Quasi-adiabatic dynamics and state preparation in Floquet many-body systems, arXiv:1611.05024.

[86] M. Eckstein and P. Werner, Damping of Bloch Oscillations in the Hubbard Model, Phys. Rev. Lett. 107, 186406 (2011).

[87] R. Peierls, On a minimum property of the free energy, Phys. Rev. 54, 918 (1938).

[88] N. N. Bogolyubov, A variation principle in the problem of many bodies, Dokl. Akad. Nauk SSSR 119, 244 (1958).

[89] R. P. Feynman, Statistical Mechanics (Benjamin, Reading, MA, 1972).

[90] M. Schüler, M. Rösner, T. O. Wehling, A. I. Lichtenstein, and M. I. Katsnelson, Optimal Hubbard Models for Materials with Nonlocal Coulomb Interactions: Graphene, Silicene, and Benzene, Phys. Rev. Lett. 111, 036601 (2013).

[91] A. Herrmann, Y. Murakami, M. Eckstein, and P. Werner, Floquet prethermalization in the resonantly driven Hubbard model, Europhys. Lett. 120, 57001 (2017). 